\title{
LAS CIUDADES Y EL DESARROLLO DE SU PAPEL INTERNACIONAL ${ }^{1}$
}

\author{
Mónica Salomón \\ Investigadora en Relaciones Internacionales \\ Facultat de Ciencies Politiques $i$ de Sociologia \\ Universitat Autònoma de Barcelona
}

Resumen

El proceso de globalización de la economía mundial de la era postindustrial ha devuelto a las ciudades el papel protagonista en el sistema económico internacional que habian perdido con el advenimiento del estado-nacion. En tanto que nexos de una economía mundial interdependiente, la actividad de las ciudades se transnacionaliza: sus vinculos con otros actores internacionales escapan cada vez más del control de los gobiernos centrales.

Por otra parte, son crecientes las demandas que diversos grupos organizados de activistas dirigen a sus gobiernos locales (cuyas competencias, tanto por razones de racionalidad econbmica como de princtpios democráticos tienden a ampliarse), exigiendo acciones o tomas de posicion de la colectividad local en temas tradicionalmente monopolizados por el estado.

Estos dos tipos de razones hacen que los estudiosos de las relaciones internacionales acepten cada vez con menos reparos a la "ciudad" (a su vez descomponible en elementos menores como gobierno local, opinión pública, grupos de interés, etc.) como integrante - -eso si, secundaria - de las taxonomias de actores internacionales.

Si al desarrollo del papel intemacional de las ciudades es un fenómeno muy reciente, atin lo es más la atención que los analistas de las relaciones internacionales le han prestado. Por el momento pueden identificarse dos tipos de enfoque. Por un lado, el de quienes intentan aconsejar a las autoridades locales sobre las estrategias que deben seguir para que sus ciudades actuien eficazmente en el marco de la economía mundial globalizada, por otro, el de quienes describen cierto tipo de actividades transnacionales emprendidas por grupos de ciudadanos en nombre de principios democráticos. Falta aún un tratamiento más abarcador, menos dirigido a sectores concretos y con una conexión más estrecha con temáticas comparables.

1. Este trabajo sintetiza parte de la memoria de licenciatura que, bajo el mismo título y dirigida por Esther Barbé, fue presenrada en la Facuirat de Ciencies Polítiques i de Sociologia de la $U A B$ en junio de 1991. 
"Papers": Revista de Sociologia

Abstract

Due to the process of globalization of the world economy in the post-industrial era, cities have recovered the major role in the international economic system which they had lost with the surging of the nation-state. As nexus of an interdependent world economy, the activities of cities become transnationalized and their links with other international actors escape more and more from the control of the central governments.

On the other hand, there are growing demands from different organized groups of activists addressed to their local governments (whose competences, for reasons related as much to economic rationality as to democratic principle, tend to widen), asking for the implementation of certain actions on behalf of the local collectivity in fields traditionally monopolized by the state.

For those two kinds of reasons, the analysts of International Relations are increasingly accepting the "city" (decomposable in its turn into smaller elements such as local government, public opinion, interest groups, etc.) as an integrant - though secondary - part of the international actors taxonomies.

If the development of the cities' international role is a recent phenomenon, even more so is the attention payed to it by the International Relations' authors. It is possible to identify two kinds of approaches. In the first place the one which seeks to advise local authorities on the best strategies in order to act effectively in the framework of the world globalized economy, in the second place the one which describes certain kind of transnational activities taken up by citizens' groups in the name of democratic principles. It is still lacking a more holistic treatment, less addressed to concrete sectors and more closely connected to other comparable subjects in the field.

Cada día somos testigos de acciones llevadas a cabo por las ciudades a nivel internacional: hermanamientos entre dos municipios de diferentes estados, conferencias internacionales de alcaldes, acuerdos comerciales gestionados por autoridades locales, redes de ciudades ricas embarcadas en proyecros de cooperación con el Tercer Mundo, creación de organizaciones de poderes locales, etc. Los ejemplos son muy numerosos y están al alcance de la mano. No cabe duda de que las ciudades adoptan un papel cada vez más dinámico en el ámbito internacional, en el que, sin cesar, cooperan, compiten, declaran, forman grupos de interés, crean su propio aparato diplomático, se promocionan o se exhiben, entre otras acciones.

En los últimos años las taxonomías de actores internacionales han empezado a dar cabida a las ciudades, que acceden al «elenco internacional", ya sea bajo el rubro de "actores gubernamentales no centrales" o del de "actores interestatales no gubernamentales" (según se ponga el énfasis en el protagonismo del poder local o en el de la colectividad). No cabe duda de que una definición abarcadora como la que preconiza García (en este mismo número) incluye sin problemas al actor "ciudad». Las ciudades, en efecto, influyen o son suscep- 
tibles de influir en la planificación de las conductas de otros actores del sistema internacional (Taylor, 1984:20).

\section{LAS CIUDADES Y EL SISTEMA INTERNACIONAL}

No deja de ser curioso el que la teoría de las relaciones internacionales haya aceptado sólo recientemente y con reticencias a este nuevo actor, dado que si bien es cierto que la capacidad de influencia de las ciudades en el sistema internacional no puede compararse con la del principal protagonista de ese sistema --el estado-, no debemos olvidar que las interacciones que mantienen las ciudades son históricamente muy anteriores a su advenimiento.

En efecto, si el estado en su versión actual no llega a los cuatro siglos de antigüedad (se suele tomar como fecha de referencia la de la Paz de Westfalia en 1648), las ciudades, en cambio, cuentan en su haber con varios milenios de historia. $Y$ desde el principio las ciudades tuvieron como función primordial la de actuar como núcleos comerciales y formar parte de sistemas de redes urbanas. Ejercieron, por lo tanto, una intensa actividad internacional.

La caída del Imperio Romano --con el consiguiente colapso en los sistemas de transporte - y la expansión del Islam en los siglos VII y VIII trajeron la crisis de la ciudad (al menos en occidente), pero la capacidad de autogobierno de las ciudades europeas se mantuvo hasta alrededor del siglo $\mathrm{XV}$. En ese sentido, una fecha significativa a este respecto es el año 1439 , año en que Enrique VI otorgó la primera carta corporativa a una municipalidad, poniendo así a las ciudades bajo el control del estado en nombre de las libertades individuales y de "la necesidad de proteger al individuo de la tiranía de los gobiernos locales y de sus prácticas corruptas» (Knight, 1989:332).

En el siglo XIX, el estado, completamente consolidado, fue capaz de dirigir a las fuerzas económicas y tecnológicas implicadas en el proceso de desarrollo industrial. Las consecuencias para las ciudades fueron enormes. En las útitimas décadas del siglo XVIi había tenido ya lugar una urbanización importante, pero hasta el siglo XIX las áreas urbanas no absorbieton una proporción amplia de la población. En el año 1800 sólo un $3 \%$ de la poblacion podía considerarse como urbana (Brunn y Williams, 1989:23). Uno de los acontecimientos más significativos del siglo XIX fue, en cambio, la concentración de la población en ciudades, concentración que prosiguió en nuestro siglo con un impulso aún mayor.

Pero si la economía de la industrialización estuvo fuertemente ligada al monopolio del poder por parte del gobierno central ( 0 , lo que para el caso es lo mismo, del estado), para la economía actual, una economía postindustrial cada vez más "globalizada", el estado y las fronteras estatales operan más bien 
como barreras, son verdaderos obstáculos a la racionalidad económica. Las ciudades, en cambio, están asumiendo un protagonismo cada vez mayor en su calidad de "nexos" de las redes económicas transnacionales. Como afirma un urbanista: "a medida que la sociedad global se expande, aumenta el papel de las ciudades y disminuye el de las naciones" (Knight, 1989:327).

El papel de las ciudades en el nuevo panorama globalizado por la rapidez y facilidad de las comunicaciones y los flujos comerciales es clave: los centros financieros, de investigación, las sedes de las empresas transnacionales y de las organizaciones internacionales están localizadas en ciudades. A la vez, las ciu* dades están cada vez más próximas unas de otras en términos de tiempo de viaje y facilidades de comunicación. Ante esta situación, los gobiemos centrales no tienen más remedio que responder a las oportunidades globales reduciendo su control y empequeñeciendo su papel en la economía, conscientes de que la economia nacional depende cada vez más de la competitividad de sus ciudades, de la calidad de las relaciones de éstas con otras ciudades y de sus vínculos globales y acceso a los centros de información.

En contrapartida, la pérdida de protagonismo del estado en los procesos de toma de decisión económica se ve acompañado de una clara extensión de la actividad estatal motivada por la necesidad de hacer frente a las demandas crecientes de la sociedad civil en el marco del estado del bienestar. Las demandas son de todo tipo y provienen de distintos grupos, organizados o no: demandas de redistribución de recursos, de mayor participación en las decisiones políticas, de asesoramiento económico, etc. Eso ha obligado a una diversificación y fragmentación en las estructuras de decisión (lo que ha conllevado, entre otras cosas, la asunción de numerosas competencias por parte de los poderes locales) y a un refinamiento considerable en los procesos de elaboración e implementación de las políticas públicas. La descentralización, junto con la elaboración de políricas públicas cada vez más refinadas y anticipatorias por gobiernos locales - particularmente en grandes ciudades - permite que las autoridades locales puedan hacer frente cada vez más eficazmente a las exigencias de proyección planteadas por la economía global y también el que puedan ejercer funciones de liderazgo y coordinación articulando demandas de diferentes grupos económicos.

No cabe duda de que las motiváciones económicas son las que más pesan en el impulso que lieva a las ciudades a actuar en la esfera internacional. Pero hay también otra dimensión en la actuación internacional de las ciudades, dimensión que, por oposición a la económica, podríamos llamar "sociopolítica". Las ciudades, en efecto, actúan también en el plano internacional por motivaciones no económicas e impulsadas por las demandas a las autoridades locales de grupos de ciudadanos que, en nombre de valores democráricos, exigen la adscripción de sus colectividades a los distintos marcos de cooperación y 
amistad internacionales o que incluso llegan a exigir a los respectivos ayuntamientos una toma de posición en cuanto a asuntos internacionales que, tradicionalmente, han sido considerados como atribuciones exclusivas del gobierno central (ver García en este mismo número).

Como ya hemos indicado, en los últimos años, los enfoques no realistas y más abiertos de las relaciones internacionales permiten dar cuenta de la actividad internacional de las ciudades. En las tipologías de actores internacionales -desde los años setenta hasta ahora - se suele incluir una categoría en la que la actividad internacional de las colectividades locales puede situarse con más o menos comodidad.

No obstante, debe decirse que, en cuanto a la caracterización y explicación del fenómeno, se ha avanzado bastante poco.

Si bien es posible identificar dos grupos de autores interesados en la actividad internacional de las ciudades - uno en su vertiente económica, el otro en la sociopolítica一, la labor que han realizado hasta la actualidad es muy poco interesante desde el punto de vista teórico. Se limita, hasta ahora, a haber comenzado la tarea meramente observacional, pretaxonómica, de identificar y agrupar actividades existentes o recomendar la ejecución de cierras actividades internacionales a las colectividades locales.

\section{LAS CIUDADES Y LAS ESTRATEGIAS DE SU INTERNACIONALIZACION}

El grupo de autores que se han interesado por dar cuenta de la actividad internacional de las ciudades en su aspecto económico-estratégico es el encabezado por P. Soldatos, quien dirige, desde 1988, el proyecto NICE (New International Cities Era), en el que participan, en su mayoría, investigadores estadounidenses y canadienses. Dicho proyecto pretende, mediante el mérodo comparativo, establecer con claridad los rasgos que hacen que una ciudad sea internacionalmente competitiva a nivel económico - que sepa enfrentarse a los nuevos desafios de la economía globalizada - para, a continuación, elaborar estrategias que permitan alcanzar el mayor grado posible de internacionalización. Como es evidente, el discurso de estos autores está dirigido a las autoridades locales y, en concreto, a los encargados de la planificación municipal.

EI discurso de NICE parte del enfoque transnacionalista de las relaciones internacionales, enfoque que--por oposición al realista, centrado en el estado y en la problemática de la seguridad militar - permite distinguir varios subsistemas dentro del sistema internacional (económico, político, cultural, etc.) y que admite la pertinencia del estudio de una gama muy amplia y variada de interacciones dentro de esos subsistemas. 
Los autores de NICE entienden que la "ciudad internacional" --con las características que reviste actualmente- es un fenómeno cuyo advenimiento se sitúa en el último cuarto de siglo y al que, a grandes rasgos, corresponde el siguiente perfil (Soldatos, 1990:27):

a) Está en una posición geográfica de apertura hacia el mundo.

b) Acoge factores de producción provenientes del extranjero (inversión y mano de obra extranjeras) y flujos comerciales (mercaderías y servicios).

c) Alberga instituciones extranjeras e internacionales (empresas, bancos y otras distintas instituciones socioeconómicas, culturales y científicas), organizaciones internacionales, comprendidas las ONG, instituciones científicas extranjeras, escuelas, universidades, etc., así como consulados y otras representaciones diplomáticas, comerciales, turísticas etc.

d) Exporta factores de producción y sus instituciones económicas (empresas, bancos, etc.), sociales, culturales y científicas están presentes en el extranjero.

e) Mantiene una multiplicidad de comunicaciones sociales con el extranjero (turísticas, postales, telefónicas, de intercambios estudiantiles, etc.).

f) Cuenta con conexiones directas con el extranjero, mediante medios de transporte y de comunicaciones en general.

g) Cuenta con un sector de servicios orientado hacia el exterior que ofrece un sistema de apoyo a los actores extranjeros o internacionales (palacios de exposiciones y congresos, hoteles, escuelas internacionales, oficinas de profesionales liberales de dimensión internacional, grandes despachos de derecho internacional, de arbitraje internacional, de contabilidad, etc.).

h) Cuenta con medios de comunicación de masas de proyección y difusión internacionales.

i) Acoge regularmente encuentros y otros tipos de actividades internacionales (congresos, exposiciones, festivales, encuentros deportivos, etc.).

j) Alberga instituciones nacionales, regionales o locales de reputación internacional o activa en el terreno de las relaciones internacionales (asociaciones y otros clubs de relaciones internacionales, grandes instituciones nacionales, especialmente socioeconómicas y científicas de alcance internacional, etc.).

k) Despliega una paradiplomacia urbana (municipal o privada) que cuenta con el apoyo de servicios especializados en relaciones internacionales. 
1) Mantiene relaciones (acuerdos, protocolos, hermanamientos, etc.) con actores extranjeros o internacionales y participa en redes internacionales de ciudades o de instituciones de ciudades.

m) La composición étnica de su población es diversificada.

Pasando ya a lo prescriptivo, Soldatos considera que una ciudad que pretenda internacionalizarse debe hacer hincapié en dos aspectos: por un lado, debe intentar atraer a la mayor cantidad posible de grandes empresas y organizaciones internacionales; por el otro, debe ser capaz de brindar un amplio abanico de servicios estratégicos de apoyo a la actividad económica de las grandes empresas: servicios financieros, de seguros, de contabilidad de gestión, comunicaciones, publicidad, etc.

Para alcanzar estos objetivos, el equipo NICE recomienda algunas acciones que las autoridades locales deberían tener en cuenta al programar la internacionalización de la ciudad:

1. Institucionalización de la paradiplomacia urbana. Esta estrategia consiste en agrupar todos los temas que tengan que ver con la actuación internacional de la ciudad bajo las competencias de una dependencia única del gobierno local, una "oficina de relaciones internacionales", que, además de organizar la actuación gubernamental, deberá ocuparse de coordinar todo tipo de iniciativas privadas.

2. Creación y refuerzo selectivo de un «tejido conjuntivo» adecuado. Las autoridades locales deberán intentar implementar una red de servicios que propicie el establecimiento - y por lo tanto atraiga - a las empresas multinacionales y a las organizaciones internacionales que más convengan a la ciudad (de acuerdo con el tipo de función que la ciudad vaya a desempeñar).

3. Selectividad de papeles ("roles»). La ciudad puede asumir distintos papeles internacionales. Se trata, pues, de discriminat cuáles de ellos podran ejercerse más eficazmente e insistir en su implementación.

4. Estrategias de alianzas bilaterales y de creación de redes. Los vínculos tradicionales entre ciudades (hermanamientos, pactos de amistad, contratos, protocolos, etc.) deben sustituirse por un nuevo concepto de alianza que suponga una racionalidad mayor.

5. Estrategia de circulos concéntricos. Esta estrategia complementa a la anterior. Se trata de insertat el desarrollo de una ciudad internacional en un esquema planificado y coherente de relaciones en sucesivos entornos (círculos): el micro-regional interno; el macro-regional interno o inter-regional; el nacional; el internacional transfronterizo; el continental y el mundial, cada uno de ellos articulado al siguiente. 
6. Socialización de las élites y de la población. Las autoridades locales deberán conseguir que se despierte una conciencia en la población en cuanto a las muchas ganancias que se pueden obtener a través de los contactos internacinales de su ciudad. Irán naciendo, así, distintos tipos de iniciativa --de contactos culturales, comerciales, científicos, etc.que las autoridades locales podrán ayudar a orientar y canalizar.

7. Liderazgo. Las autoridades locales no sólo deben canalizar demandas, sino también asumir un papel conductor en las diferentes iniciativas de internacionalización.

8. Marketing urbano e image-building. Es importante dar, hacia el exterior, una imagen de "internacionalidad" que atraiga a empresas y organizaciones internacionales. El proceso de internacionalización tiene, pues, mucho de retroalimentación: cuanto más internacionalizada sea la imagen que se tiene de la ciudad, más facilidades tendrá para internacionalizarse.

De hecho, el trabajo del grupo de autores encabezados por Soldatos parte de la descripción de acciones ya emprendidas por algunas ciudades, ciudades que estarían a la vanguardia en cuanto a su internacionalización (con una mayoría de los rasgos de los arriba mencionados) y que, por ello, merecen ser tomadas como modelos por el resto. Creemos que es interesante describir con algún detalle el ejemplo de la puesta en práctica de una estrategia de internacionalización municipal.

\section{LA CIUDAD DE SEATTLE Y SU OFICINA DE ASUNTOS INTERNACIONALES ${ }^{2}$}

Quizás la estrategia más calurosamente recomendada por el equipo NICE, sea la de la "creación de una paradiplomacia urbana». La insistencia en que funcione una instancia de coordinación de la actividad internacional de la ciudad dependiente de la autoridad local es comprensible, dado que la existencia de un núcleo organizativo constituye el prerrequisito de prácticamente todas las demás estrategias.

Se trata de una iniciativa que en los últimos años han tomado muchas ciudades, sobre todo estadounidenses. Fry (1990:7) indica que son más de doscientas las ciudades de los EE.UU. que han establecido oficinas dedicadas al

2. La información necesaria paza la tedacción de este apareado nos ha sido proporcionada por la propia OLA, que visitamos en el mes de agosto de 1990 . Agradecemos al Sr. Keith Orton clentonces Director en funciones de la OIA - tanto la entrevista que nos concedió como el material proporcionado. 
comercio internacional, a la inversión, al turismo y a los intercambios culturales. Son cada vez más numerosos los gobiernos locales que intentan racionalizar sus actividades internacionales estableciendo una oficina de coordinación, a menudo producto de la fusión de distintas dependencias.

Numerosos autores consideran que la Oficina de Asuntos Internacionales (Office of International Affairs, en adelante OIA), de la ciudad estadounidense de Seattle es un modelo digno de estudio y de imitación (Fry, 1990; Cohn e.a., 1989), sobre todo porque su creación obedeció a una muy cuidadosa ponderación de las demandas y necesidades existentes en la ciudad en cuanto a organización de la actividad internacional, de acuerdo con una orientación claramente "anticipatoria" y muy de acuerdo con lo que preconizan los distintos enfoques de elaboración de políticas públicas (y, desde luego, el equipo NICE).

Las características geográficas, demográficas y económicas de Seattle permiten afirmar que presenta un claro "perfil internacional", según la definición de NICE.

Su emplazamiento geográfico es el ideal para establecer contactos comerciales. Ubicada en el estado de Washington (extremo noroeste de los EEUU), a $182 \mathrm{~km}$ de la frontera de EE.UU. con Canadá y a igual distancia de Londres que de Tokio, Seattle se está consolidando como punto clave para el desarrollo del comercio internacional de los EE.UU. Unas excelentes infraestructuras de comunicación - puerto, aeropuerto, red viaria- facilitan las conexiones.

La próspera economía de la ciudad (Seattle está clasificada en diversas encuestas como una de las ciudades del mundo que disfruta de un nivel de vida más elevado) tiene una base diversificada. El comercio internacional y el turismo tienen en ella un gran papel, pero también la empresa Boeing, que da trabajo a más de noventa mil personas, cubriendo la mitad de los puestos de trabajo en manufacturas.

La población de Seattle (500.000 habitantes en el área urbana y l,1 millones en la región) es mulciétnica y multicultural (con un $10 \%$ de asiáticos) y alcanza uno de los niveles educativos más altos de los EE.UU. La ciudad está bien dotada de centros culturales y recreativos.

Numerosas empresas extranjeras - asiáticas, europeas y australianas - tienen sede en la ciudad, así como veintisiete consulados extranjeros.

La OIA, instancia coordinadora de la actividad internacional de Seattle, fue creada el i de septiembre de 1986, como parte del Departamento Ejecutivo del Ayuntamiento de la ciudad y con la finalidad explícita de "controlar las responsabilidades internacionales municipales, $y$ ofrecer apoyo al comercio internacional, turismo y desarrollo económico» (OlA, 1989). Su staffes reducido: un director, dos coordinadores técnicos y un funcionario administrativo. El director es elegido por el alcalde, quien, a su vez, elige a los dos coordina- 
dores técnicos. El presupuesto (alrededor de 230.000 dólares anuales) cubre casi exclusivamente los salarios del staff.

Antes de la creación de la OIA, la infraestructura existente a nivel municipal para canalizar los contactos internacionales estaba muy poco coordinada. Intervenían en ella dos instancias diferentes: el Departamento de Desarrollo Comunitario y la Oficina de Relaciones Intergubernamentales. La oficina del alcalde también mantenía correspondencia sobre comercio internacional y debía ocuparse de las entrevistas y visitas def alcalde relacionadas con asuntos incluídos en esa temática. A la dispersión de las actividades internacionales de la municipalidad se sumaba la falta de recursos presupuestarios necesarios para cubrir diversas actividades concernientes a su proyección internacional (programa de hermanamientos, programas culturales, visitantes).

La conciencia de esas carencias impulsó al entonces alcalde de Seattle a encargar un estudio sobre la posible creación de una instancia coordinadora de la actividad internacional, a la consultoría Arthur Young. Ésta, en base a una serie de entrevistas con funcionarios municipales y otros actores comunitarios, identificó tres series de datos básicos (Malachuk y Brandon, 1986):

a) Una serie de metas percibidas por la comunidad en lo referente al papel internacional de la ciudad; una de ellas era, naturalmente, el reforzamiento de la actividad económica de la ciudad haciéndola asumir el papel de centro de negocios internacional y de comercio mundial. Pero la comunidad de Seattle manifestaba también interés en enriquecer y ampliar sus oportunidades educativas y culturales y expresar su consenso en temas relacionados con la justicia internacional y los derechos humanos.

b) Distintas áreas de énfasis: servicios relacionados con el comercio internacional (de transporte, financieros, etc.), convenciones y conferencia, turismo, inversión exterior, exportaciones locales, protocolo, dirección y supervisión de políticas y programas de hermanamientos ${ }^{3}$, entre otros.

c) Los grupos de interés implicados en la proyección internacional de la ciudad, que inclúán, entre otros, a la administración portuaria, grupos comerciales, compañías privadas, agencias gubernamentales, cámara de comercio, comités de hermanamientos de ciudades, escuelas y universidades, activistas locales e iglesias.

3. Los hermanamientos son vínculos de intercambio y cooperación incernacional entre dos comunidades establecidas a través de sus gobiernos locales y formalizados mediante una declaracion conjunta. Seattle mantiene vínculos de hermanamiento con catorce ciudades: Bergen (Noruega), Tashkent (ex-URSS), Beersheva (Israel), Mazatlán (México), Nantes (Francia), Mombasa (Kenia), Christchurch (Nueva Zelanda), Chongqing (China), Limbe (Camerún), Managua (Nicaragua), Reykjavik (Islandia), Galway (Irlanda) y Taejon (Corea del Sur). 
Con estos datos, los autores del informe recomendaron un diseño de OLA que, dados los recursos disponibles del ayuntamiento y otros grupos de interés comunitario, y los puntos de vista de las diferentes partes implicadas, debia dedicarse fundamentalmente a las áreas de apoyo al comercio internacional, protocolo y hermanamientos de ciudades y, en menor grado, en la organización de convenciones, turismo, promoción de exportaciones y programas culturales.

Las indicaciones del informe fueron seguidas casi al pie de la letra. Muy poco después de su presentación, la OlA abrió sus puertas.

Mencionaremos algunas de las actividades que ha llevado a cabo en sus principales áreas de actuación:

Hermanamientos. A la OIA corresponde asesorar a los comités sobre posibles actividades, protocolo (cómo se debe recibir a la delegación extranjera) y también canaliza el proceso de formalización del vínculo de hermanamiento. La coordinadora de la OIA de programas comunitarios se reúne con los delegados de los catorce comités para discutir los programas y las maneras de llevarlos a cabo. El Ayuntamiento de Seattle, además, ha establecido unos criterios a los que deben ajustarse las peticiones de establecimiento de hermanamientos (amplia base de apoyo ciudadano, presupuesto, plan de actividades, etc.).

Las actividades a que han dado lugar los vínculos de Seattle con sus catorce ciudades hermanas son múltiples. Incluyen intercambios de estudiantes de enseñanza media y profesionales, visitas de delegaciones culturales (artistas, músicos, etc.), ayudas a orfanatos, compericiones deportivas, etc.

Actividades de promoción de exportaciones y turismo. Consisten, fundamentalmente, en el apoyo a los organismos existentes en la ciudad. Como ejemplos de esa asistencia puede citarse la ayuda para el establecimiento de un "Instituto de Comercio Internacional" en un "Community College» local, la asistencia a los comités de hermanamiento en asuntos relacionados con el comercio y el apoyo a distintas organizaciones de la ciudad encargadas de promover las exportaciones: Cámara de Comercio, Washington Council for International Trade, Washington-China Relation Council, Japan-Americ Society.

En el ámbito del turismo, la OIA coopera con el puerto y la "Seattle-King County Convention and visitors Bureau for tourism promotion", de la que han nacido diversos programas de misiones comerciales al exterior, conferencias de alcaldes, etc.

Protocolo. La OIA se ocupa de la organización de la recepción de los visitantes internacionales y de los viajes al extranjero del alcalde y de las 
"Papers": Revista de Sociologia

misiones comerciales. A menudo un rerpresentante de la OIA participa en estos viajes.

Tanto el staff de la OIA como la autoridades municipales y los representantes de los grupos de interés relacionados con ella, coinciden en describir su funcionamiento como óptimo.

\section{LA «POLÍTICA EXTERIOR MUNICIPAL»}

El segundo enfoque en la consideración de la actividad internacional de las ciudades, desde la disciplina de las Relaciones Internacionales, está representado por la obra de Chadwick $(1977,1990)$, cuyo discurso está dirigido, sobre todo, a fomentar una mayor participación ciudadana a través del gobierno local en un área tradicionalmente reservada a los gobiernos centrales: la política exterior. Los receptores del discurso no son los planificadores urbanos, sino los líderes locales de distintos movimiento ciudadanos. Su planteamiento constituye una crítica al estado como institución y llega a preconizar la sustitución del estado como unidad de análisis en las relaciones internacionales por la de ciudad, en una postura más de militante que de teórico ${ }^{4}$ : el estado, en efecto, sigue siendo un actor importantísimo en el sistema internacional, por más que podamos discutir su exclusividad y durabilidad dentro del mismo. Por otra partc, es evidente que las acciones de las ciudades, por más que interesantes, pintorescas y cada vez más numerosas, afectan al menos por ahora poco a los demás actores del sistema.

De todos modos, creemos que no está de más pasar revista a algunas de las acciones que han emprendido determinadas colectividades urbanas, actuando muchas veces en contra de las directrices de la política exterior estatal.

Una de estas actividades es la de «desnuclearización municipal». Consiste en la formalización de una declaración que prohibe toda actividad nuclear en los límites del municipio (Newcombe \& Newcombe, 1988:52). El movimiento, iniciado por las ciudades japonesas de Handa, Minato y Mishima en protesta contra los ensayos nucleares en el Océano Pacífico, no trascendió las fronteras japonesas hasta varias décadas después. En Europa el movimiento de desnuclearización se desarrolló en los ochenta, a partir de la resolución adoptada por la municipalidad de Manchester. En la actualidad, Japón continúa a la cabeza por el número de municipios desnuclearizados (1215) y España, con más de cuatrocientos, ocupa el segundo lugar (Fisas, 1988). Numerosos municipios de Gran Bretaña, Holanda, Bélgica, Italia y Alemania se han uni-

4. Esto se explica — si no se justifica - por la doble condición de tebrico y de activiša de Alger, que fue secretario de la IPRA (Internarional Pcace Research Association). 
do con entusiasmo al movimiento. Las ciudades de Canadá y Nueva Zelanda son también muy activas en este terreno.

Es especialmente interesante la envergadura que ha tomado el movimiento de desnuclearización municipal entre las ciudades estadounidenses. En 1990 había unos ciento setenta municipios desnuclearizados en la primera potencia nuclear del mundo. Mientras que en otros estados las declaraciones son más bien simbólicas y pretenden más que nada contribuir a crear una opinión pública a favor del desarme, en los EE.UU. los ayuntamientos desnuclearizados afectan directamente a la poderosa industria armamentística del país, que se ha visto en numerosas ocasiones obligada a cambiar los emplazamientos de sus instalaciones. Numerosas campañas llevadas a cabo por los grupos de fabricantes de armas contra resoluciones de desnuclearización, muestran que sus efectos empiezan a ser algo más que meramente simbólicos.

Los ayuntamientos de EE.UU. y Canadá son particularmente activos en cuanto a la adopción de resoluciones municipales tras la aprobación de referéndums sugeridos por grupos de activistas locales.

Numerosos ayuntamientos estadounidenses han aprobado resoluciones sobre "desinversión": la retirada de inversiones de la corporación municipal en empresas relacionadas de alguna manera con la industria de armamento. Otras resoluciones están referidas a la reconversión de industrias armamentísticas locales para evitar, tras su desaparición, un desempleo masivo. La desinversión es también una acción que emprenden los ayuntamientos en pro del respeto a los derechos humanos. En 1990, la corte surpema del estado de Maryland declaró legales las iniciativas de más de cien jurisdicciones locales que habían desinvertido los fondos de pensiones a su cargo en compañías con tratos comerciales con África del Sur como represalia contra el apartheid (MFP 1990/4:4).

La ciudad de Seattle fue muy activa en cuanto a resoluciones municipales relacionadas con la protección de los derechos humanos y en pro de la paz mundial hasta hace pocos años. La conflictiva América Central, en particular, fue objeto de numerosas declaraciones impulsadas por grupos de activistas. Sus contenidos iban desde solicitar al gobierno federal el cese de ayuda a la "Contra" hasta el de decidir desobedecer la normativa estatal en cuanto a inmigrantes para proteger a los centroamericanos refugiados, a quienes las autoridades negaban estatuto de refugiados, e incluso la creación de comisión municipal de ayuda a Centroamérica. Pero, meses después, una resolución en sentido opuesto, aprobada por iniciativa de un grupo de ciudadanos de tendencia conservadora, prohibió al municipio ocuparse de temas considerados de ámbito exclusivo del estado.

Por último, en la lista de resoluciones municipales de carácter internacionalista cabe incluir el tipo de declaración define a la ciudad en cuestión es una 
"ciudad mundial». El movimiento de "mundialización» fue iniciado en Japón en 1950 y fue seguido por Francia, pocos años después. La declaración supone un reconocimiento de los problemas globales (amenaza nuclear, polución ambiental, etc.) y la necesidad de una organización supranacional capaz de darles solución (Newcombe \& Newcombe, 1988:10). Generalmente las ciudades declaradas mundializadas reúnen aportaciones para contribuir a los programas multilaterales de cooperación al desarrollo. En la actualidad hay casi mil localidades del mundo que se han declarado mundializadas Francia (quinientas) y Japón (trescientas) reúnen a la mayor parte; el resto se reparte entre la RFA, Reino Unido, Dinamarca, Bélgica, Canadá, Italia y los EE.UU.

\section{CONCLUSION}

En estas páginas apenas hernos presentado una muestra de lo que constituyen las múltiples y diversificadas acciones que las ciudades están llevando a cabo a nivel internacional, en particular en el subsistema económico. No cabe duda de que las ciudades son un actor internacional cada vez más consistente y organizado 5 . Las autoridades locales, por su parte, asumen cada vez mayores responsabilidades en el liderazgo del proceso de internacionalización. El ejemplo de Seattle en el diseño y ejecución de una estrategia innovadora da buenas muestras de eilo.

En cuanto a la consideración que las ciudades han recibido en la disciplina de Relaciones Internacionales, constatamos que con ellas ocurre lo mismo que con otros actores no estatales: la atención que han recibido hasta el momento es aún muy escasa. Por otra parte, tanto la línea utransnacionalista» de NICE como la de los estudiosos provenientes del área de la peace research dirigen sus respectivos discursos hacia destinatarios muy concretos: en un caso a los planificadores urbanos que necesitan "recetas" para proyectar sus ciudades hacia el ámbito internacional, en el otro a los grupos de activistas que dedican suts esfuerzos a la consecución de la paz mundial. Se echa en falta un discurso más general y más imbricado con una teoría general de las Relaciones Internacionales.

5. No obsrante, en determinados casos pensamos que puede tener más eficacia teórica el caraccerizar a la ciudad no como "actorn sino como issue area (área temática) en la que interactúan distintos actores: opinión pública, organizaciones gubernamenzales, ONGs, gobiemo local, etc. 
Las ciudades y el desarrollo de su papel internacional

\section{BIBLLOGRAFÍA CITADA}

Alger, Ch. (1977), "The impact of cities on international systems", Ekistics, 264, nov. 1977.

Alger, Ch. (1990), "The World Relations of Cities: Closing the Gap Berween Social Science Paradigms and Everyday Human Experience», International Studies Quaterly, 1990, 34, pp. 493-518.

Brunn, S.D. y Williams, J.F. (1983), Cities of the World: World Regional Urban Development, New York, Harper \& Row.

Bulletin of Municipal Foreign Policy (trimestral), Irvine (California), Center for $I_{n-}$ novative Diplomacy.

Fisas Armengol, V. (1984), El desarme en casa. Municipios desnuclearizados y desarme regional, Barcelona, Fontamara.

Fisas Armengol, V. (1988), Los municipios y la paz, Barcelona, CIDOB.

Fry, E. H. (1989), "International Cities in the United States: Economic Dimensions». Ponencia presentada en la conferencia Processus d'Internationalisation des Villes, Centre Jacques Cartiet, Lyon, Francia, 6-7 dic. 1989.

Ery, E., Radebaugh, L.H. y Soldatos, P. (1989), The New International Cities Era: The Global Activities of North American Municipal Governments, Provoh (Utah), Brigham Young University.

Knighr, R.V. y Gappert, G. (eds.) (1989), Cities in a Global Society, Urban Affairs Annual Reviews, London, Sage Publication.

Knight, R.V. (1989), "The Emergent Global Sociery", en Knight y Gapperr (eds.), pp. $24-57$.

Knight, R.V. (1989), "Ciry Building in a Global Society", en Knight y Gappert (eds.), pp. 326-345.

Malachuk, D. y Brandon, J. (1986), An Organizational Assessment of the Proposed Office of International Affairs, Seatrle, Arthur Young.

Newcombe, A. y Newcombe, H. (1988), "Peace Action at the Municipal Level", Peace Research Reviews, vol. XI, núm. 2, sept. 1988.

Office of International Affairs (City of Seattle) (1989), Seattle Datasheet.

Soldatos, P. (1990), La nouvelle génération de villes internationales dans un monde transnationalisé, La Dimensión internacional de las ciudades, seminario de la UIMP, Valencia, 25-29 junio 1990.

Taylor, Ph. (1984), Nonstate actors in International Politics. From transregional to Substate Organizations, Boulder, Westview Press. 